\title{
CENTRAL LIMIT THEOREM FOR ZERO-RANGE PROCESSES*
}

\author{
Jeremy QuAstel $^{\dagger}{ }^{\dagger}$ Hanna Jankowski ${ }^{\dagger}$ And John SherifF $^{\dagger}$
}

Abstract. We consider additive functionals $\int_{0}^{t} V\left(\eta_{s}\right) d s$ of symmetric zero-range processes, where $V$ is a mean zero local function. In dimensions 1 and 2 we obtain a central limit theorem for $a^{-1}(t) \int_{0}^{t} V\left(\eta_{s}\right) d s$ with $a(t)=\sqrt{t \log t}$ in $d=2$ and $a(t)=t^{3 / 4}$ in $d=1$ and an explicit form for the asymptotic variance $\sigma^{2}$. The transient case $d \geq 3$ can be handled by standard arguments $[\mathrm{KV}, \mathrm{SX}, \mathrm{S}]$. We also obtain corresponding invariance principles. This generalizes results obtained by Port (see [CG]) for noninteracting random walks and Kipnis [K] for the symmetric simple exclusion process. Our main tools are the martingale method together with $L^{2}$ decay estimates [JLQY] for the process semigroup.

0. Introduction. In a system of interacting random walks on $\mathbb{Z}^{d}$ one may consider a local function $V$ and its time integral $\int_{0}^{t} V\left(\eta_{s}\right) d s$. The total number of particles is a conserved quantity and correspondingly such a system will typically have a one parameter family of invariant measures $\nu_{\rho}$ parametrized by the average particle density $\rho$. If particle number is the only conserved quantity the system is ergodic and, if we start with density $\rho$ then the time integral $\int_{0}^{t} V\left(\eta_{s}\right) d s$ will converge to the appropriate expectation $E_{\nu_{\rho}}[V]$.

Next it is natural to ask about the fluctuations of $\int_{0}^{t}\left(V\left(\eta_{s}\right)-E_{\nu_{\rho}}[V]\right) d s$. We observe them under the stationary process $P_{\rho}$ obtained by starting the process with the invariant measure $\nu_{\rho}$ with density $\rho$. A computation (see $[\mathrm{CG}]$ ) shows that for independent symmetric nearest neighbour random walks, with each particle jumping at rate $2 d$, and $V=\eta(0)$, the number of particles at the origin,

$$
\frac{1}{a(t, d)} \int_{0}^{t}\left(\eta_{s}(0)-\rho\right) d s
$$

converges in law to a normal distribution with mean zero and variance $\sigma^{2}(d, \rho)$ where

$$
\begin{aligned}
& d=1: a(t, d)=t^{3 / 4} \\
& d=2: a(t, d)=\sqrt{t \log t} \\
& d \geq 3: a(t, d)=\sqrt{t} .
\end{aligned}
$$

and $\sigma^{2}(1, \rho)=4 \rho / 3 \sqrt{\pi}, \sigma^{2}(2, \rho)=\rho / 2 \pi$, and $\sigma^{2}(d, \rho)=2 \rho \int_{0}^{\infty} p_{t}(0,0) d t$ in $d \geq 3$, where $p_{t}(x, y)$ are the transition probabilities of the corresponding continuous time random walk. The recurrence of the individual particles is responsible for the unusual scaling in one and two dimensions.

Kipnis $[\mathrm{K}]$ showed that the analogous results hold for symmetric random walks with simple exclusion, with a new variance given by $\sigma^{2}(1, \rho)=4 \rho(1-\rho) / 3 \sqrt{\pi}$, $\sigma^{2}(2, \rho)=\rho(1-\rho) / 2 \pi$ if $d=2$, and $\sigma^{2}(d, \rho)=2 \rho(1-\rho) \int_{0}^{\infty} p_{t}(0,0) d t$ in $d \geq 3$. However, these results are somewhat special as they rely on explicit calculations. In particular, they rely heavily on the special self-duality of symmetric simple exlusions

* Received July 20, 2001; accepted for publication January 10, 2003.

$\dagger$ Department of Mathematics, University of Toronto, Toronto, Ontario, Canada M5S 3G3 (quastel@math.toronto.edu); (jankowski@utstat.toronto.edu); (sheriff@utstat.toronto.edu). Partially supported by NSERC. 
from which the equation $(\lambda-L) G_{\lambda}(\eta)=\eta(0)-\rho$ is explicity solved by $G_{\lambda}(\eta)=$ $\sum_{x} g_{\lambda}(x)(\eta(x)-\rho)$ where $g_{\lambda}(x)=\int_{0}^{\infty} e^{-\lambda t} p_{t}(0, x) d t$ solves $(\lambda-\Delta) g_{\lambda}=\delta(0)$ for the lattice Laplacian $\Delta f(x)=\sum_{y \sim x}(f(y)-f(x))$. For more complicated $V$ one can in principle use duality to obtain analogous results. However, these are still very special exact computations and one can ask whether something more general is available. Here we are interested in the zero-range models; systems of continuous-time random walks where each particle's jump rate is affected by the total number of particles at its site. No duality is available, and unless special assumptions are imposed, the models are not attractive. However they do have an explicit family $\nu_{\rho}$ of (product) invariant measures.

In the transient case, $d \geq 3$, one can appeal to general results of Kipnis and Varadhan $[\mathrm{KV}]$ for additive functionals of reversible Markov processes which apply whenever we have a bound $E\left[\left(\int_{0}^{t} V\left(\eta_{s}\right) d s\right)^{2}\right] \leq C t, V$ mean zero. More precisely, they show that the central limit theorem holds for $t^{-1 / 2} \int_{0}^{t} V\left(\eta_{s}\right) d s$ whenever the asymptotic variance, $-2 E\left[V L^{-1} V\right]$, is finite. In $d \geq 3$ this holds for any bounded local function. This still leaves open the cases $d=1,2$ where typically $-2 E\left[V L^{-1} V\right]=\infty$. For symmetric simple exclusion and zero-range models, it is known [SX] that under mild conditions, $-2 E\left[V L^{-1} V\right]<\infty$ if and only if $\bar{V}^{(n)}(\rho)=0$ for $n=0,1,2$ in $d=1$, $n=0,1$ in $d=2$ and $n=0$ in $d \geq 3$ where

$$
\bar{V}(\rho)=E_{\nu_{\rho}}[V]
$$

and $\bar{V}^{(n)}$ denotes the $n$th derivative.

Recently it was shown [JQLY] that under mild assumptions, the $L^{2}$ decay of a mean zero function $V$ to equilibrium is at rate $t^{-d / 2}$ for the symmetric zero-range dynamics. More precisely,

$$
E_{\nu_{\rho}}\left[\left(P_{t} V\right)^{2}\right]=C(\rho, d)\left[\bar{V}^{\prime}(\rho)\right]^{2} t^{-d / 2}+o\left(t^{-d / 2}\right)
$$

with an explicit constant $C(\rho, d)$. $P_{t}$ here denotes the semigroup $e^{t L}$ of the dynamics. In other words, up to leading order, the only contribution of a local function $V$ is through its (formal) projection onto $\sum_{x}\left(\eta_{x}-\rho\right)$, and all other details are lost.

From (0.3) one obtains immediately that the variance $E\left[\left(\frac{1}{a(t, d)} \int_{0}^{t} V\left(\eta_{s}\right) d s\right)^{2}\right] \rightarrow$ $\tilde{C}(\rho, d)\left[\bar{V}^{\prime}(\rho)\right]^{2}$ in $d=1$ and 2 with $a(t, d)$ as before; the unusual factors $a(t)=t^{3 / 4}$ in $d=1$ and $a(t)=\sqrt{t \log t}$ in $d=2$ simply compensate for the non-integrability of the correlations $t^{-d / 2}$. But also the fact that most of the details of $V$ are lost means that one can replace $V$ by more or less any local function, and a central limit theorem for one would imply the same for the other. Hence our strategy is to find a nice local function $f$ for which the martingale method used by Kipnis goes through, i.e. for which we can solve $(\lambda-L) G_{\lambda}=f$ more or less explicitly, and then use (0.3) to extend it to a general local function $V$.

In Section 6 we consider the symmetric simple exclusion process. Because of the special self-duality property, (0.3) can be obtained easily. In fact one can compute the next order term. Hence for symmetric simple exclusion in one dimension we can study the fluctuations of $\int_{0}^{t} V\left(\eta_{s}\right) d s$ under $P_{\rho}$ when $V(\rho)=V^{\prime}(\rho)=0$. The scaling turns out to be $a(t, 2)$ and the limit is normal with variance $C\left[V^{\prime \prime}(\rho)\right]^{2}$ with an explicit constant $C$. An interesting open problem is whether the corresponding result can be obtained for the zero-range models. 
Although one expects a result analogous to (0.3) for a large class of systems, at present it is only known for symmetric zero-range and simple exclusion processes (where one can use duality to give a complete expansion). For the important case of the Ising model with Kawasaki dynamics, even under strong mixing conditions, (0.3) is open and the best that is available at this time is a bound of the form $t^{-d / 2}(\log t)^{\alpha}$ for some $\alpha>0$ [LQY]. It would also be very interesting to obtain related results for asymmetric systems. Only partial results are available $[\mathrm{S}]$.

Finally, we note that our methods give full invariance principles, i.e., process limits of $X_{t}^{N}=\frac{1}{a(N, d)} \int_{0}^{N t} V\left(\eta_{s}\right) d s$. Of course $X_{t}^{N}$ by itself is not a Markov process. Using the martingale method we can obtain that the limit is Gaussian. Since

$$
\lim _{N \rightarrow \infty} \frac{1}{N \log N} \int_{0}^{N t_{2}} \int_{0}^{N t_{1}}\left(1+\left|s_{2}-s_{1}\right|\right)^{-1} d s_{1} d s_{2}=2 \min \left(t_{1}, t_{2}\right)
$$

and

$$
\lim _{N \rightarrow \infty} \frac{1}{N^{3 / 2}} \int_{0}^{N t_{2}} \int_{0}^{N t_{1}}\left(1+\left|s_{2}-s_{1}\right|\right)^{-1 / 2} d s_{1} d s_{2}=\frac{4}{3}\left[t_{2}^{3 / 2}+t_{1}^{3 / 2}-\left|t_{2}-t_{1}\right|^{3 / 2}\right],
$$

we see from (0.3) that the asymptotic covariance is $4 C(\rho, d)\left[\bar{V}^{\prime}(\rho)\right]^{2} \min \left(t_{1}, t_{2}\right)$ in $d=2$ and $\frac{4 \sqrt{2}}{3} C(\rho, d)\left[\bar{V}^{\prime}(\rho)\right]^{2}\left[t_{2}^{3 / 2}+t_{1}^{3 / 2}-\left|t_{2}-t_{1}\right|^{3 / 2}\right]$ in $d=1$. Hence in $d=2($ and $d \geq 3)$ the limit is Brownian motion, while in $d=1$ the limit is a fractional Brownian motion with exponent $3 / 2$. In particular, the Markov property is recovered in the limit in $d \geq 2$ but not in $d=1$.

\section{Notation and Results.}

Zero-range model. Particles are distributed on the lattice $\mathbb{Z}^{d}$, with $\eta(x)$ denoting the number of particles at site $x \in \mathbb{Z}^{d}$. Configurations will be called $\eta$ and the state space is the set $\mathbb{N}^{d}$ of such configurations. We also choose jump rates $c: \mathbb{N} \rightarrow \mathbb{R}_{+}$such that $c(0)=0<c(k)$ for $k \geq 1$. The dynamics of the process is described as follows. If there are $\eta(x)$ particles at site $x$ then the rate at which a particle jumps from $x$ to nearest neighbour site $y$ is $c(\eta(x))$. In other words, each particle at $x$ jumps at rate $2 d c(\eta(x)) / \eta(x)$. When it jumps it chooses at random from its $2 d$ neighbours. This takes place independently of all the other particles, and the new configuration $\eta^{x, y}$ obtained from $\eta$ in this way is given by $\eta^{x, y}(z)=\eta(z)+\delta(z, y)-\delta(x, z)$ where $\delta(x, y)=1$ if $x=y$ and 0 otherwise.

The dynamics we have described is a Markov process on the state space $\mathbb{N}^{d}$ whose generator acts on functions that depend only on a finite number of coordinates as

$$
L f(\eta)=\sum_{x \sim y} c(\eta(x))\left[f\left(\eta^{x, y}\right)-f(\eta)\right]
$$

where $x \sim y$ denotes (ordered) nearest neighbours.

To ensure that the process is well defined and also to guarantee that the system on a box of side length $N$ has spectral gap of order $N^{-2}$ (see [LSV]), we assume that for some $B<\infty-B \leq c(n+1)-c(n) \leq B$ for all $n=0,1, \ldots$ and that there exists $\delta>0$ and $k_{0} \geq 1$ such that $c(m)-c(n) \geq \delta$ for all $m-n \geq k_{0}$. Note that it follows from the assumptions that for some $\kappa<\infty$, for all $n=0,1, \ldots, \kappa^{-1} n \leq c(n) \leq \kappa n$.

Denote by $Z: \mathbb{R}_{+} \rightarrow \mathbb{R}_{+}$the partition function defined by $Z(\varphi)=\sum_{k \geq 0} \varphi^{k} / c(k)$ ! where $c(k) !=c(1) \cdots c(k)$. Note that from $\kappa^{-1} n \leq c(n)$ the radius of convergence of 
$Z$ is infinite. The dynamics we have described conserves the total number of particles and the product measure $\nu$ on $\mathbb{N}^{d}$ with marginals

$$
\nu\left(\eta_{x}=j\right)=[Z(\varphi)]^{-1} \varphi^{j} / c(j) !
$$

for $j \in \mathbb{N}, x \in \mathbb{Z}^{d}$, for $0 \leq \varphi<\infty$ represent a full set of extremal translation invariant, invariant measures $[\mathrm{A}]$. Let $\rho(\varphi)=E_{\bar{\nu}_{\varphi}}\left[\eta_{0}\right]$ be the density of particles for the measure $\bar{\nu}_{\varphi} . \quad \rho:[0, \infty) \rightarrow[0, \infty)$ is a smooth strictly increasing bijection. Since $\rho(\varphi)$ has a physical meaning as the density of particles, instead of parameterizing the above family of measures by $\varphi$, we use the density $\rho$ as parameter and we write $\nu_{\rho}$, $\rho \in[0, \infty)$ for the corresponding product measures. Note $\varphi$ is a smooth function whose derivative is bounded above and below by a strictly positive constant on each compact set of $\mathbb{R}_{+}($cf. $[\mathrm{KL}])$. Note that $\rho=E_{\nu_{\rho}}[\eta(0)]=\varphi Z^{\prime}(\varphi) / Z(\varphi)$ and

$$
E_{\nu_{\rho}}\left[c\left(\eta_{x}\right)\right]=\varphi(\rho)
$$

The process is reversible with respect to each $\nu_{\rho}$, i.e. the generator $L$ is symmetric on $L^{2}\left(\nu_{\rho}\right)$. Fix a density $\rho>0$ and denote by $P_{\rho}$ the corresponding stationary process with marginals $\nu_{\rho}$.

A function $V: \mathbb{N}^{\mathbb{Z}^{d}} \rightarrow \mathbb{R}$ is called local if it only depends on $\eta(x), x \in \Lambda$ where $\Lambda$ is a finite subset of $\mathbb{Z}^{d}$.

We will say that a local function $V$, depending on $\eta_{x},|x| \leq R$, has a polynomial bound if for some $N, C_{1}, C_{2}<\infty$,

$$
|V(\eta)| \leq C_{1}+C_{2}\left[\sum_{|x| \leq R} \eta(x)\right]^{N}
$$

In $[J L Q Y]$ it is shown that $(0.3)$ holds for bounded local functions with

$$
C(d, \rho)=\chi(\rho)\left(8 \pi \varphi^{\prime}(\rho)\right)^{-d / 2} .
$$

We will prove below it holds for any local function with polynomial bound. Note that in our model

$$
\chi(\rho)=\operatorname{Var}_{\nu_{\rho}}(\eta(0))=\varphi(\rho) / \varphi^{\prime}(\rho)
$$

THEOREM 1.1. Consider zero-range models satisfying the above conditions. Let $V$ be a mean zero local function with polynomial bound, and consider

$$
X_{t}^{N}=\frac{1}{a(N, d)} \int_{0}^{N t} V\left(\eta_{s}\right) d s
$$

where $a(t, d)$ is given by (0.1). Under $P_{\rho}, X_{t}^{N} \Rightarrow X_{t}$ where $X_{t}$ is

$d=1$ : fractional Brownian motion,

$$
\operatorname{cov} \cdot \frac{2 \varphi(\rho)}{3 \sqrt{\pi}\left|\varphi^{\prime}(\rho)\right|^{3 / 2}}\left[\bar{V}^{\prime}(\rho)\right]^{2}\left[t_{2}^{3 / 2}+t_{1}^{3 / 2}-\left|t_{2}-t_{1}\right|^{3 / 2}\right],
$$

$d=2:$ Brownian motion, covariance $\frac{\varphi(\rho)}{2 \pi\left|\varphi^{\prime}(\rho)\right|^{2}}\left[\bar{V}^{\prime}(\rho)\right]^{2} \min \left(t_{1}, t_{2}\right)$,

$d \geq 3$ : Brownian motion, covariance $2\left\langle V(-L)^{-1} V\right\rangle \min \left(t_{1}, t_{2}\right)$.

The case $d \geq 3$ follows from the general central limit theorem for additive functionals of reversible Markov processes $[\mathrm{KV}]$ together with the fact $[\mathrm{SX}]$ that in $d \geq 3$ 
every mean zero local function with polynomial bound has $\left\langle V(-L)^{-1} V\right\rangle\langle\infty$ (which also follows easily from the decay estimate). The $d=2$ case will be proved in sections 2 and 3 and the $d=1$ case will be proved in sections 2 and 4 .

Symmetric simple exclusion. Particles are performing symmetric continuous time random walks on the lattice $\mathbb{Z}^{d}$ with the exclusion rule that jumps to already occupied sites are suppressed. If we start with at most one particle per site then it remains so for all time. The state space is then $\{0,1\}^{\mathbb{Z}^{d}}$ and the generator acts on local functions as

$$
L f(\eta)=\sum_{x \sim y}\left(f\left(\eta^{x y}\right)-f(\eta)\right)
$$

where $\eta^{x y}$ denotes the configuration obtained from $\eta$ by switching the occupation numbers at $x$ and $y$. For simplicity we have considered the nearest neighbour case with jump rate one to each nearest neighbour site: The results will all have straightforward generalizations to any symmetric finite range jump law. The invariant measures of the process are the product measures $\nu_{\rho}$ with $\nu_{\rho}\left(\eta_{x}=1\right)=\rho, \rho \in[0,1]$. These are also reversible. Let $P_{\rho}$ denote the stationary process with initial distribution $\nu_{\rho}$.

TheORem 1.2 . Consider the symmetric simple exclusion process described above. $i$. Let $V$ be a mean zero local function, and consider

$$
X_{t}^{N}=\frac{1}{a(N, d)} \int_{0}^{N t} V\left(\eta_{s}\right) d s
$$

where $a(t, d)$ is given by (0.1). Under $P_{\rho}, X_{t}^{N} \Rightarrow X_{t}$ where $X_{t}$ is

$d=1:$ fractional Brownian motion, cov. $\frac{2 \rho(1-\rho)}{3 \sqrt{\pi}}\left[\bar{V}^{\prime}(\rho)\right]^{2}\left[t_{2}^{3 / 2}+t_{1}^{3 / 2}-\left|t_{2}-t_{1}\right|^{3 / 2}\right]$,

$d=2:$ Brownian motion, covariance $\frac{\rho(1-\rho)}{2 \pi}\left[\bar{V}^{\prime}(\rho)\right]^{2} \min \left(t_{1}, t_{2}\right)$,

$d \geq 3$ : Brownian motion, covariance $2\left\langle V(-L)^{-1} V\right\rangle \min \left(t_{1}, t_{2}\right)$.

ii. In $d=1$, let $V$ be a local function with $\bar{V}(\rho)=\bar{V}^{\prime}(\rho)=0$. Let

$$
X_{t}^{N}=\frac{1}{\sqrt{N \log N}} \int_{0}^{N t} V\left(\eta_{s}\right) d s .
$$

Under $P_{\rho}, X_{t}^{N} \Rightarrow$ Brownian motion, covariance $\frac{\rho^{2}(1-\rho)^{2}}{8 \pi}\left|\bar{V}^{\prime \prime}(\rho)\right|^{2} \min \left(t_{1}, t_{2}\right)$.

Part $i$ was proved in $[\mathrm{K}]$ for $V(\eta)=\eta_{0}-\rho$ (note that our process has been sped up by a factor $2 d$ relative to his). The case $d \geq 3$ follows from [KV] and [SX] as before. $d \leq 2$ is proved in an analogous way to Theorem 1.1, using the analogous decay estimate (0.3) which can be obtained easily for symmetric simple exclusion using duality. Part $i i$ is proved in Section 6 by using duality to obtain the next term in the decay estimate.

2. $L^{2}$ decay to equilibrium. The main tool used to prove Theorem 1.1 is the following result adapted from [JLQY]. Let $P_{t}$ denote the semigroup $e^{t L}$.

Theorem 2.1. Let $V$ be a local function with polynomial bound (1.4) and $E_{\nu_{\rho}}[V]=0$. Then there exists $\gamma>0$ and $R<$ infty and depending only on $d$ 
and $C_{1}, C_{2}, N$ appearing in (1.4) such that

$$
\left|t^{d / 2} E_{\nu_{\rho}}\left[\left(P_{t} V\right)^{2}\right]-C(\rho, d)\left[\bar{V}^{\prime}(\rho)\right]^{2}\right| \leq R t^{-\gamma}
$$

holds with $C(\rho, d)$ given by (1.5).

Proof. As stated in [JLQY], (0.3) holds only for bounded $V$. Keeping careful track through that proof of the dependence of the $o\left(t^{-d / 2}\right)$ term on $\|V\|_{\infty}$ we obtain the following statement: There exists an $\epsilon>0$ and a constant $K(d, \rho)<\infty$ such that for any local function $V$, for all $t \geq 1$,

$$
\left|t^{d / 2} E_{\nu_{\rho}}\left[\left(P_{t} V\right)^{2}\right]-C(d, \rho)\left[\bar{V}^{\prime}(\rho)\right]^{2}\right| \leq K(d, \rho)\left[\|V\|_{\infty}^{2}\right] t^{-\epsilon} .
$$

For $V$ satisfying (1.4) we write $V=W_{t}+X_{t}$ where $W_{t}=V \wedge t^{\epsilon / 4}$ and $X_{t}=$ $\left(V-V \wedge t^{\epsilon / 4}\right)$. By the contractivity in $L^{2}$ of $P_{t}$, and $\left|X_{t}\right| \leq f 1_{f>t^{\epsilon / 4}}$ where $f=$ $C_{1}+C_{2}\left[\sum_{|x| \leq R} \eta(x)\right]^{N}$, and by the Schwarz inequality, we have $E_{\nu_{\rho}}\left[\left(P_{t} X_{t}\right)^{2}\right] \leq$ $\|f\|_{L^{4}}^{1 / 2}\left[P\left(f \geq t^{\epsilon / 4}\right)\right]^{1 / 2}$ which is exponentially small in $t$. Also $\left|\bar{W}_{t}^{\prime}(\rho)-\bar{V}^{\prime}(\rho)\right| \leq$ $E_{\nu_{\rho}}\left[f 1_{f>t^{\epsilon / 4}} \sum_{x}\left(\eta_{x}-\rho\right)\right] \leq c t^{-a}$ for some $c<\infty$ and $a>0$ depending only on $C_{1}, C_{2}$ and $N$. Applying (2.2) to $W_{t}$ the result follows.

The proof of the following lemma can be found in [SV].

Lemma 2.2 . (Garsia, Rodemich, Rumsey) Let $p$ and $\Psi$ be continuous, strictly increasing functions on $[0, \infty)$ such that $p(0)=\Psi(0)=0$ and $\lim _{t \rightarrow \infty} \Psi(t)=$ $\infty$. Given $T>0$ and $X_{t} \in C([0, T], \mathbb{R})$, then for $0 \leq \tau \leq T-\delta$,

$$
\left|X_{\tau+\delta}-X_{\tau}\right| \leq 8 \int_{0}^{\delta} \Psi^{-1}\left(\frac{4}{u^{2}} \int_{0}^{T} \int_{0}^{T} \Psi\left(\frac{\left|X_{t}-X_{s}\right|}{p(|t-s|)}\right) d s d t\right) d p(u) .
$$

Corollary 2.3 . Let $P_{N}$ be probability measures on $C([0, T], \mathbb{R})$ satisfying

$$
E_{P_{N}}\left[\left(X_{t}-X_{s}\right)^{2}\right]=C(N)|t-s|^{1+\gamma}
$$

for $t, s \in[0, T]$ with $C(N) \leq C<\infty$ and independent of $t$ and $s$, and $\gamma>0$. Then $P_{N}$ are tight. If $C(N) \rightarrow 0$ as $N \rightarrow \infty$, then $P_{N}$ converge weakly to the trivial process concentrated on $X_{t} \equiv 0$.

Proof. We apply the lemma of Garsia, Rodemich, and Rumsey with $\Psi(x)=x^{2}$. After an application of Schwarz's inequality we obtain

$$
E_{P_{N}}\left[\sup _{|t-s| \leq \delta}\left|X_{t}-X_{s}\right|\right] \leq 16 \int_{0}^{\delta} \frac{d p(u)}{u}\left(\int_{0}^{T} \int_{0}^{T} \frac{E_{P_{N}}\left[\left|X_{t}-X_{s}\right|^{2}\right]}{p(|t-s|)^{2}} d s d t\right)^{1 / 2} .
$$

From (2.3) if we choose $p(x)=x^{\alpha}$ with $1+\frac{\gamma}{2}>\alpha>1$, the right hand side is bounded by $A \sqrt{C(N)} \delta^{\alpha-1}$ for some $A<\infty$. This proves that $P_{N}$ are tight. If $C(N) \rightarrow 0$ it is clear that $X_{t} \equiv 0$ is the only possible limit.

COROllary 2.4 . Let $d \leq 2$. Let $V$ be a local function satisfying (1.4) with

$$
\bar{V}^{\prime}(\rho)=\bar{V}(\rho)=0 .
$$


Let $P_{N}$ be the distribution of $X_{t}^{N}=\frac{1}{a(N, d)} \int_{0}^{N t} V\left(\eta_{s}\right) d s, t \in[0, T]$ under $P_{\rho}$. Then $P_{N}$ converge weakly to the trivial process concentrated on $X_{t} \equiv 0$..

Proof. By Fubini's theorem,

$$
E\left[\left(\int_{0}^{t} V\left(\eta_{s}\right) d s\right)^{2}\right]=2 \int_{0}^{t} \int_{0}^{s_{2}} E_{\nu_{\rho}}\left[V P_{s_{2}-s_{1}} V\right] d s_{1} d s_{2} .
$$

By the reversibility of the process this is equal to

$$
2 \int_{0}^{t} \int_{0}^{s_{2}} E_{\nu_{\rho}}\left[\left(P_{\frac{s_{2}-s_{1}}{2}} V\right)^{2}\right] d s_{1} d s_{2}
$$

By the decay estimate this becomes, in $d=1$ or 2 ,

$$
a^{2}(d, t) C_{1}\left[\bar{V}^{\prime}(\rho)\right]^{2} \chi(\rho)\left(8 \pi \varphi^{\prime}(\rho)\right)^{-d / 2}+C_{2} t^{2-\frac{d}{2}-\gamma},
$$

for $C_{1}, C_{2}<\infty$ depending only on $\rho$ and $d$. Since $\bar{V}^{\prime}(\rho)=0$ the result follows from the previous corollary.

In the sections 4 and 5 we will prove the following result.

Lemma 2.5 . Let

$$
X_{t}^{N}=\frac{1}{a(N, d)} \int_{0}^{N t}\left(c\left(\eta_{s}\right)-\varphi(\rho)\right) d s .
$$

Let $P_{N}$ be the distribution of $X_{t}^{N}$ under $P_{\rho}$. Then $P_{N}$ are tight and has unique weak limit: In $d=1$ fractional Brownian motion of parameter $3 / 2$ with covariance $\frac{2 \varphi(\rho) \sqrt{\varphi^{\prime}(\rho)}}{3 \sqrt{\pi}}\left[t_{2}^{3 / 2}+t_{1}^{3 / 2}-\left|t_{2}-t_{1}\right|^{3 / 2}\right] ;$ In $d=2$, Brownian motion with covariance $\frac{\varphi(\rho)}{2 \pi} \min \left(t_{1}, t_{2}\right)$.

Proof of theorem 1.1. We can write

$\int_{0}^{t} V\left(\eta_{s}\right) d s=\int_{0}^{t}\left(V\left(\eta_{s}\right)-\frac{\bar{V}^{\prime}(\rho)}{\varphi^{\prime}(\rho)}\left[c\left(\eta_{s}(0)\right)-\varphi(\rho)\right]\right) d s+\frac{\bar{V}^{\prime}(\rho)}{\varphi^{\prime}(\rho)} \int_{0}^{t}\left[c\left(\eta_{s}(0)\right)-\varphi(\rho)\right] d s$.

By Corollary 2.3 the first term, suitably rescaled, is tight and converges to the trivial process $X_{t} \equiv 0$. By the previous lemma, the second term is tight and converges to the limit in theorem 1.1.

3. Some coefficients from potential theory. We now compute some explicit constants which play a role in the limiting variance. Let $p_{t}(x, y)$ be the solution of

$$
\frac{\partial p}{\partial t}=\Delta p, \quad p_{0}(x, y)=\delta(x-y)
$$

where $\Delta f_{x}=\sum_{y \sim x} f_{y}-f_{x}$ is the lattice Laplacian and $\delta(x)$ takes the value 1 at $x \in \mathbb{Z}^{d}$ and 0 otherwise. Note that $p_{t}(x, y)=e^{-2 d t} \sum_{n=0}^{\infty} \frac{(2 d t)^{n}}{n !} p_{n}(x, y)$ where $p_{n}(x, y)$ are the transition probabilities of a symmetric simple random walk on $\mathbb{Z}^{d}$. We have, as $t \rightarrow \infty$,

$$
p_{t}(0,0) \sim(4 \pi(1+t))^{-d / 2} .
$$

For each $\lambda>0$, let

$$
g_{\lambda}(x)=\int_{0}^{\infty} e^{-\lambda t} p_{t}(0, x) d t
$$


$x \in \mathbb{Z}^{d}$, so that

$$
(\lambda-\Delta) g_{\lambda}=\delta(0)
$$

Lemma 3.1 . i. In $d=2, \lim _{\lambda \rightarrow 0} \frac{1}{-\log \lambda} \sum_{x \sim y}\left(g_{\lambda}(y)-g_{\lambda}(x)\right)^{2}=1 / 2 \pi$,

ii. In $d \leq 2,\left\|g_{\lambda}\right\|_{\ell^{2}\left(\mathbb{Z}^{d}\right)}^{2}=\frac{1}{2} \int_{0}^{\infty} t e^{-\lambda t} p_{t}(0,0) d t \sim \lambda^{\frac{d}{2}-2}$.

Proof.

$i i$ follows from (3.1). Multiplying (3.2) by $g_{\lambda}$ and summing gives $\lambda \sum_{x}\left[g_{\lambda}(x)\right]^{2}+$ $\frac{1}{2} \sum_{x \sim y}\left(g_{\lambda}(y)-g_{\lambda}(x)\right)^{2}=g_{\lambda}(0)$. By ii, $\lim _{\lambda \rightarrow 0} \frac{1}{-\log \lambda} \sum_{x \sim y}\left(g_{\lambda}(y)-g_{\lambda}(x)\right)^{2}=$ $2 \lim _{\lambda \rightarrow 0} \frac{1}{-\log \lambda} g_{\lambda}(0)$. Now use (3.1) again.

Let

$$
v_{t}(x)=\int_{0}^{t} p_{s}(0, x) d s
$$

so that

$$
\frac{\partial v}{\partial t}=\Delta v+\delta(0), \quad v_{0}(x)=0 .
$$

Lemma 3.2 . In $d=1, i . \lim _{t \rightarrow \infty} t^{-3 / 2} \sum_{x}\left[v_{t}(x)\right]^{2}=\frac{4}{3 \sqrt{\pi}}(\sqrt{2}-1)$,

ii. $\lim _{t \rightarrow \infty} t^{-3 / 2} \int_{0}^{t} \sum_{x \sim y}\left[v_{s}(y)-v_{s}(x)\right]^{2} d s=\frac{4}{3 \sqrt{\pi}}(2-\sqrt{2})$.

Proof

i. $\sum_{x}\left[v_{t}(x)\right]^{2}=\sum_{x} \int_{0}^{t} \int_{0}^{t} p_{s_{1}}(0, x) p_{s_{2}}(0, x) d s_{1} d s_{2}=2 \int_{0}^{t} \int_{0}^{s_{2}} p_{s_{1}+s_{2}}(0,0) d s_{1} d s_{2}$. Using (3.1) we obtain $i$. To obtain $i$, multiply (3.3) by $v$ and integrate to obtain $\int_{0}^{t} \sum_{x \sim y}\left[v_{s}(y)-v_{s}(x)\right]^{2} d s=2 \int_{0}^{t} v_{s}(0) d s-\sum_{x}\left[v_{t}(x)\right]^{2}$ and use (3.1) again.

4. Martingale method $(\mathbf{d}=2)$. In this section we prove Lemma 2.5 in dimension $d=2$ using the martingale method. Let

$$
G_{\lambda}(\eta)=\sum_{x \in \mathbb{Z}^{d}} g_{\lambda}(x)(\eta(x)-\rho), \quad \hat{G}_{\lambda}(\eta)=\sum_{x \in \mathbb{Z}^{d}} g_{\lambda}(x)(c(\eta(x))-\varphi(\rho)) .
$$

A summation by parts yields

$$
L G_{\lambda}(\eta)=c(\eta(0))-\varphi(\rho)-\lambda \hat{G}_{\lambda}(\eta) .
$$

Hence we can write

$$
\frac{1}{\sqrt{N \log N}} \int_{0}^{N t}\left(c\left(\eta_{s}(0)\right)-\varphi(\rho)\right) d s=-M_{t}^{N}+R_{t}^{N}
$$

where

$$
M_{t}^{N}=\frac{1}{\sqrt{N \log N}}\left[G_{1 / N}\left(\eta_{N t}\right)-G_{1 / N}\left(\eta_{0}\right)-\int_{0}^{N t} L G_{1 / N}\left(\eta_{s}\right) d s\right]
$$

is a martingale and

$$
R_{t}^{N}=\frac{1}{\sqrt{N \log N}}\left[G_{1 / N}\left(\eta_{N t}\right)-G_{1 / N}\left(\eta_{0}\right)+\frac{1}{N} \int_{0}^{N t} \hat{G}_{1 / N}\left(\eta_{s}\right) d s\right] .
$$

We compute

$\left\|G_{\lambda}\right\|_{L^{2}\left(\nu_{\rho}\right)}^{2}=\operatorname{Var}_{\nu_{\rho}}(\eta(0))\left\|g_{\lambda}\right\|_{\ell^{2}\left(\mathbb{Z}^{d}\right)}^{2}$ and $\left\|\hat{G}_{\lambda}\right\|_{L^{2}\left(\nu_{\rho}\right)}^{2}=\operatorname{Var}_{\nu_{\rho}}(c(\eta(0)))\left\|g_{\lambda}\right\|_{\ell^{2}\left(\mathbb{Z}^{d}\right)}^{2}$. From lemma 3.1 it follows that $E_{P_{\rho}}\left[\left(R_{t}^{N}\right)^{2}\right] \leq C t / \log N$. Hence, once we show $R_{t}^{N}$ is tight, 
it will converge to the trivial process $X_{t} \equiv 0$. This is done at the end of this section in Lemma 4.2 .

For $M_{t}^{N}$ we use the central limit theorem for martingales $[\mathrm{R}]$ in the following form.

LEMma 4.1. If $M_{t}^{N}$ are martingales satisfying $i .\left\langle M^{N}\right\rangle(t) \rightarrow \sigma^{2} t$ in probability, $t \in[0, T] ;$ ii. $\sigma_{\epsilon}\left(M^{N}\right)(t)=\sum_{0 \leq \tau_{i} \leq t}\left|\Delta M_{N}\left(\tau_{i}\right)\right|^{2} 1\left(\left|\Delta M_{N}\left(\tau_{i}\right)\right| \geq \epsilon\right) \rightarrow 0$ in probability, for each $\epsilon>0$. Then $M_{t}^{N}$ conveges to a Brownian motion with variance $\sigma^{2}$. Here $\langle M\rangle(t)$ is the variance process of a martingale $M(t)$, defined by $M^{2}(t)-\langle M\rangle(t)$ is a martingale, and $\tau_{i}$ are the jump times, and $\Delta M\left(\tau_{i}\right)$ the jumps of the process.

Note that if $M_{t}=G\left(t, \eta_{t}\right)-G\left(0, \eta_{0}\right)-\int_{0}^{t}\left\{\partial_{u}+L\right\} G\left(u, \eta_{u}\right) d u$ then $E\left[\left(M_{t}-\right.\right.$ $\left.\left.M_{s}\right)^{2} \mid \mathcal{F}_{s}\right]=E\left[\int_{s}^{t} \sum_{x \sim y} c\left(\eta_{x}\right)\left(\nabla_{x y} G\right)^{2} d u \mid \mathcal{F}_{s}\right]$ where $\nabla_{x y} G(\eta)=G\left(\eta^{x y}\right)-G(\eta)$. In our situation, $\nabla_{x y} G_{\lambda}(\eta)=g_{\lambda}(y)-g_{\lambda}(x)$. Hence we can compute by Lemma 3.1.i.,

$$
\left\langle M^{N}\right\rangle(t)=\frac{\varphi(\rho) t}{\log N} \sum_{x \sim y}\left(g_{1 / N}(y)-g_{1 / N}(x)\right)^{2} \rightarrow \frac{\varphi(\rho) t}{2 \pi},
$$

which gives $i$ of Lemma 4.1. To check $i i$, note that

$\sigma_{\epsilon}\left(M^{N}\right)(t)=\frac{1}{\log N} \sum_{x \sim y} N^{-1} P_{x y}^{N}(t)\left(g_{1 / N}(y)-g_{1 / N}(x)\right)^{2} \mathbf{1}\left(g_{1 / N}(y)-g_{1 / N}(x) \geq \sqrt{N \log N} \epsilon\right)$

where $P_{x y}^{N}$ are independent Poisson processes running at rate $N \eta(x)(1-\eta(y)$. This converges to 0 as $N \rightarrow \infty$ by the law of large numbers and another application of Lemma 3.1.i.

The following lemma completes the proof of Theorem 1 in $d=2$.

Lemma 4.2 . The family of processes $R_{t}^{N}$ defined in (4.2) is tight.

Proof. Note first that $\frac{1}{\sqrt{N \log N}}\left[G_{1 / N}\left(\eta_{N t}\right)-G_{1 / N}\left(\eta_{0}\right)\right]=J_{t}^{N}+M_{t}^{N}$ where $J_{t}^{N}=$ $\frac{1}{\sqrt{N \log N}} \int_{0}^{N t} L G_{1 / N}\left(\eta_{s}\right) d s$ and $M_{t}^{N}$ is tight. From the Feynman-Kac formula and the variational formula for the principal eigenvalue of $L+V$,

$$
\frac{1}{2 t} \log E\left[\exp \left\{\alpha \int_{0}^{t} L G_{1 / N}\left(\eta_{s}\right) d s\right\}\right] \leq \sup _{E_{\nu}[f]=1, f \geq 0}\left\{\alpha E_{\nu}\left[L G_{1 / N}(\eta) f(\eta)\right]+E_{\nu}[\sqrt{f} L \sqrt{f}]\right\} .
$$

Now $E_{\nu}\left[L G_{1 / N} f\right]=\frac{1}{2} \sum_{x \sim y} E_{\nu}\left[c(\eta(x))\left(f\left(\eta^{x y}\right)-f(\eta)\right)\left(g_{1 / N}(y)-g_{1 / N}(x)\right)\right]$ and $E_{\nu}[f L f]=$ $\frac{1}{2} \sum_{x \sim y} E_{\nu}\left[c(\eta(x))\left(f\left(\eta^{x y}\right)-f(\eta)\right)^{2}\right]$. By Schwarz's inequality and $f\left(\eta^{x y}\right)-f(\eta)=$ $\left(\sqrt{f}\left(\eta^{x y}\right)+\sqrt{f}(\eta)\right)\left(\sqrt{f}\left(\eta^{x y}\right)-\sqrt{f}(\eta)\right)$ the supremum is bounded by $C \alpha^{2} \sum_{x \sim y}\left(g_{1 / N}(y)-\right.$ $\left.g_{1 / N}(x)\right)^{2}$. By stationarity and Lemma 3.1 we obtain,

$$
E\left[\exp \left\{\alpha\left(J_{t}^{N}-J_{s}^{N}\right)\right\}\right] \leq \exp \left\{C(N) \alpha^{2}|t-s|\right\} .
$$

In fact $C(N)=O\left(N^{-1}\right)$, but for compactness we only need that $C$ is bounded. Applying Lemma 2.2 with $\Psi(x)=e^{x}-1$ and $p(x)=\sqrt{x}$ after some computation one obtains from (4.3) that for some $C(T)<\infty$,

$$
E\left[\sup _{\substack{0 \leq s<t \leq T \\|t-s| \leq \delta}}\left|J_{t}^{N}-J_{s}^{N}\right|\right] \leq C \sqrt{\delta}(1+\log \delta),
$$

which gives the compactness of $J_{t}^{N}$. 
Now we can write $R_{t}^{N}=A_{t}^{N}+B_{t}^{N}+C_{t}^{N}$ where $A_{t}^{N}=\frac{1}{\varphi^{\prime} N \sqrt{N \log N}} \int_{0}^{N t}\left[G_{1 / N}\left(\eta_{N t}\right)-\right.$ $\left.G_{1 / N}\left(\eta_{s}\right)\right] d s, B_{t}^{N}=-\frac{1}{N \sqrt{N \log N}} \int_{0}^{N t}\left[\hat{G}_{1 / N}\left(\eta_{s}\right)-\frac{1}{\varphi^{\prime}} G_{1 / N}\left(\eta_{s}\right)\right] d s$ and $C_{t}^{N}=\frac{1}{\sqrt{N \log N}}[(1-$ $\left.\left.\frac{t}{\varphi^{\prime}}\right) G_{1 / N}\left(\eta_{N t}\right)-G_{1 / N}\left(\eta_{0}\right)\right] . A_{t}^{N}$ and $C_{t}^{N}$ are tight by the previous argument. $B_{t}^{N}$ is tight by Corollaries 2.3 and 2.4 .

5. Martingale method $(\mathbf{d}=1)$. In this section we prove Lemma 2.5 for zerorange models on the one dimensional integer lattice. Using the decay estimate Theorem 2.1, and a variance computation (such as in the proof of Corollary 2.4), we can see that (2.3) is satisfied for $X_{t}^{N}=\frac{1}{a(N, 1)} \int_{0}^{N t}\left(c\left(\eta_{s}\right)-\varphi(\rho)\right) d s$ for some $C(N)=C<\infty$. Hence $X_{t}^{N}$ are tight. Furthermore the asymptotic covariance can be computed by (0.5). Therefore it only remains to show that the limiting process is Gaussian.

Note that $u_{t}(x)=\int_{0}^{\varphi^{\prime}(\rho) t} p_{s}(0, x) d s$ is the solution of

$$
\partial_{t} u=\varphi^{\prime}(\rho)\left[\Delta u+\delta_{0}\right], \quad u(x, 0) \equiv 0 .
$$

Let

$$
U_{t}^{T}(\eta)=\sum_{x} u_{T-t}(x)(\eta(x)-\rho) .
$$

Then $M_{t}^{T}=U_{t}^{T}\left(\eta_{t}\right)-U_{0}^{T}\left(\eta_{0}\right)-\int_{0}^{t}\left(\partial_{s}+L\right) U_{s}^{T}\left(\eta_{s}\right) d s$ is a martingale in $t$ up to time $T$, and by explicit computation of $\left(\partial_{s}+L\right) U_{s}^{T}$ we have

$$
\int_{0}^{T}\left(c\left(\eta_{t}(0)\right)-\varphi(\rho)\right) d t=M_{T}^{T}+U_{0}^{T}\left(\eta_{0}\right)+R_{T}
$$

where

$$
R_{T}=\int_{0}^{T} \sum_{x} q_{T-t}(x) a\left(\eta_{t}(x)\right) d s,
$$

where $a(n)=c(n)-\varphi(\rho)-\varphi^{\prime}(\rho)(n-\rho)$, and $q_{t}(x)=p_{\varphi^{\prime}(\rho) t}(0, x)$. In particular,

$$
\frac{1}{N^{3 / 4}} \int_{0}^{N T}\left(c\left(\eta_{s}(0)\right)-\varphi(\rho)\right) d s=\frac{1}{N^{3 / 4}} M_{N T}^{N T}+\frac{1}{N^{3 / 4}} U_{N T}^{N T}\left(\eta_{0}\right)+\frac{1}{N^{3 / 4}} R_{N T} .
$$

We first show that the error term $N^{-3 / 4} R_{N T}$ is negligible. We write the expectation of its square as

$$
\frac{1}{N^{3 / 2}} \int_{0}^{N T} \int_{0}^{N T} \sum_{x_{1}, x_{2}} q_{N T-s_{1}}\left(x_{1}\right) q_{N T-s_{2}}\left(x_{2}\right) E\left[a\left(\eta_{s_{1}}\left(x_{1}\right)\right) a\left(\eta_{s_{2}}\left(x_{2}\right)\right)\right] d s_{1} d s_{2} .
$$

Since we have a stationary Markov process the expectation can be written as $E_{\mu_{\rho}}\left[a_{x_{1}} P_{\left|s_{2}-s_{1}\right|} a_{x_{2}}\right]$ where we use $a_{x}$ to denote the function $\eta \mapsto a(\eta(x))$. By the Markov property, and reversibility of the process this can be rewritten as

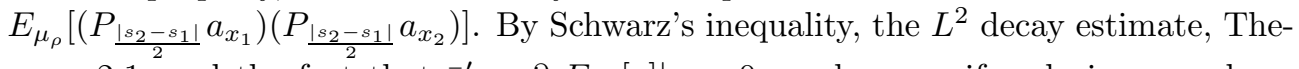
orem 2.1, and the fact that $\bar{a}^{\prime}=\left.\partial_{q} E_{\mu_{q}}[a]\right|_{\rho}=0$, we have, uniformly in $x_{1}$ and $x_{2}$, $E\left[a\left(\eta_{s_{1}}\left(x_{1}\right)\right) a\left(\eta_{s_{2}}\left(x_{2}\right)\right)\right]=o\left(\left|s_{2}-s_{1}\right|^{-1 / 2-\gamma}\right)$ for some $\gamma>0$. The summations over $x_{i}$ of $q .\left(x_{i}\right)$ are each 1 , and hence, performing the two time integrals, after a simple change of variables we obtain for some $\alpha>0$.

$$
E\left[\left(N^{-3 / 4} R_{N T}\right)^{2}\right]=O\left(N^{-\alpha}\right) .
$$


Now note that $U_{0}^{T}\left(\eta_{0}\right) \in \mathcal{F}_{0}$ and $M_{T}^{T}$ are independent. By the central limit theorem, $N^{-3 / 4} U_{0}^{N T}\left(\eta_{0}\right)$ converges to a Gaussian process. Note that

$$
M_{t}^{T}=\sum_{x \sim y} \int_{0}^{t} \nabla_{x y} U_{s}^{T} d P_{s}(x, y)
$$

where $P_{t}^{x y}$ are independent, compensated Poisson martingales jumping at rate $c\left(\eta_{s}(x)\right)$. Since $\nabla_{x y} U_{s}^{T}=u_{T-s}(y)-u_{T-s}(x)$ we have

$$
\begin{aligned}
\frac{1}{N^{3 / 4}} M_{N T}^{N T}= & \frac{1}{N^{3 / 4}} \sum_{x \sim y} \int_{0}^{N T}\left[u_{N T-s}(y)-u_{N T-s}(x)\right] d P_{s}(x, y) \\
& =\frac{1}{\sqrt{N}} \sum_{\substack{x \in \mathbb{Z} / \sqrt{N} \\
e= \pm 1}} \int_{0}^{T} v_{T-s}^{N, e}(x) d Q_{s}^{N, e}(x) .
\end{aligned}
$$

where

$$
\begin{aligned}
& v_{t}^{N, e}(x)=\left[u_{N t}(\sqrt{N} x+e)-u_{N t}(\sqrt{N} x)\right], \\
& Q_{t}^{N, e}(x)=N^{-1 / 4} P_{N t}(\sqrt{N} x, \sqrt{N} x+e) .
\end{aligned}
$$

Now $\sum_{x \in \mathbb{Z} / \sqrt{N}} Q_{t}^{N, e}(x) \delta_{x}$ converges to a space-time white noise $\sqrt{\varphi(\rho)} \dot{W}(t, x)$ (with variance $\varphi(\rho)$ ). We can identify $v_{t}^{N, e}(x)$ with a function on $\mathbb{R}$ by, say, polygonalizing between values of $\mathbb{Z} / \sqrt{N}$. Then by local central limit theorem $[\mathrm{L}], v_{t}^{N, e}(x) \rightarrow$ $\operatorname{sgn}(e) v_{t}(x)$ strongly, where $v_{t}(x)=\int_{0}^{t} x e^{-x^{2} / 4 s} s^{-3 / 2} \frac{d s}{4 \sqrt{\pi}}$. Therefore $\frac{1}{N^{3 / 4}} M_{N T}^{N T}$ converges to the Gaussian process given by the sum of two independent copies (for $e= \pm 1$ ) of

$$
\sqrt{\varphi(\rho)} \int_{\mathbb{R}} \int_{0}^{T} v_{t}(x) \dot{W}(d t, d x) .
$$

This completes the proof of Theorem 1 in $d=1$.

6. Symmetric simple exclusion. Symmetric simple exclusion possesses a very special self-dual property, which can be expressed in several different ways. For our purposes we use the following description. Fix $\rho \in(0,1)$ and for each finite subset $A$ of $\mathbb{Z}^{d}$ let

$$
\tilde{\eta}_{A}=\prod_{x \in A} \frac{\eta_{x}-\rho}{\sqrt{\chi(\rho)}}
$$

where in this model

$$
\chi(\rho)=\rho(1-\rho) .
$$

The collection $\tilde{\eta}_{A}, A$ a finite subset of $\mathbb{Z}^{d}$, is an orthonormal basis of $L^{2}\left(\pi_{\rho}\right)$. Let $E_{n}$ denote the span of $\tilde{\eta}_{A},|A|=n$. Then $L^{2}\left(\pi_{\rho}\right)$ is the direct sum of the $E_{n}$. The special self-dual property of symmetric simple exclusion is that $L: E_{n} \rightarrow E_{n}$. In particular,

$$
L \tilde{\eta}_{A}=\sum_{B \sim A}\left(\tilde{\eta}_{B}-\tilde{\eta}_{A}\right)
$$

where $B \sim A$ means that $B$ can be obtained from $A$ by deleting a site from $A$ and appending its nearest neighbour. If $f \in L^{2}\left(\pi_{\rho}\right)$ then we have $f=\sum_{n=0}^{\infty} \sum_{|A|=n} \tilde{f}_{A} \tilde{\eta}_{A}$ 
and $L f=\sum_{n=0}^{\infty} \sum_{|A|=n} \tilde{L}_{n} \tilde{f}_{A} \tilde{\eta}_{A}$ where

$$
\tilde{L}_{n} \tilde{f}_{A}=\sum_{B \sim A}\left(\tilde{f}_{B}-\tilde{f}_{A}\right) .
$$

In other words, on each $E_{n}$, the process can be identified with a continuous time random walk $A_{t}$ on the subsets of $\mathbb{Z}^{d}$ of cardinality $n$, where jumps to all nearest neighbour sets take place at rate one. Let us denote the corresponding semigroup $P_{t}^{(n)} \tilde{f}_{A}=E_{A}\left[\tilde{f}_{A_{t}}\right]$.

The duality allows one to make rather explicit computations. In particular, we can compute the next order term in (0.3).

TheOrem 6.1 . Let $P_{t} V(\eta)=E_{\eta}\left[V\left(\eta_{t}\right)\right]$ be the semigroup of symmetric simple exclusion with generator (1.8). For any local function with $E_{\nu_{\rho}}[V]=0$,

$$
\begin{array}{lll}
d=1 & : & E_{\nu_{\rho}}\left[\left(P_{t} V\right)^{2}\right]=\chi(\rho)(8 \pi)^{-1 / 2}\left[\bar{V}^{\prime}(\rho)\right]^{2} t^{-1 / 2}+\frac{\chi(\rho)^{2}}{32 \pi}\left|\bar{V}^{\prime \prime}(\rho)\right|^{2} t^{-1}+O\left(t^{-3 / 2}\right) \\
d=2 & : & E_{\nu_{\rho}}\left[\left(P_{t} V\right)^{2}\right]=\chi(\rho)(8 \pi)^{-1}\left[\bar{V}^{\prime}(\rho)\right]^{2} t^{-1}+a_{2}(\rho, V) t^{-2}+O\left(t^{-3}\right) \\
d \geq 3 & : & E_{\nu_{\rho}}\left[\left(P_{t} V\right)^{2}\right]=\chi(\rho)(8 \pi)^{-d / 2}\left[\bar{V}^{\prime}(\rho)\right]^{2} t^{-d / 2}+a_{d}(\rho, V) t^{-(d+2) / 2}+O\left(t^{-d}\right),
\end{array}
$$

where

$$
\begin{aligned}
& a_{2}(\rho, V)=\frac{\chi(\rho)^{2}}{128 \pi^{2}}\left|\bar{V}^{\prime \prime}(\rho)\right|^{2}+\frac{1}{128 \pi}\left|\bar{V}^{\prime}(\rho)\right|^{2}-\frac{1}{16 \pi}\left[\sum_{j=1}^{2} \sum_{x}\left|x_{j}\right|^{2} \tilde{V}_{x}+\left(\sum_{x} x_{j} \tilde{V}_{x}\right)^{2}\right] \\
& a_{d}(\rho, V)=-\frac{1}{(8 \pi)^{d / 2}}\left[\frac{1}{2} \sum_{j=1}^{d} \sum_{x}\left|x_{j}\right|^{2} \tilde{V}_{x}+\frac{1}{2}\left(\sum_{x} x_{j} \tilde{V}_{x}\right)^{2}+2^{-5}\left(d^{2}-3 d\right)\left|\bar{V}^{\prime}(\rho)\right|^{2}\right], \quad d \geq 3
\end{aligned}
$$

Remark. The next term in the expansion seems to have no meaning except in $d=1$.

Proof. From the Parseval relation and the duality described above

$$
E_{\nu_{\rho}}\left[\left(P_{t} V\right)^{2}\right]=\sum_{n=1}^{\infty} \sum_{|A|=n}\left[P_{t}^{(n)} \tilde{V}_{A}\right]^{2} .
$$

Since $P_{t}^{(n)}$ corresponds to random walk on an orthant of $\left(\mathbb{Z}^{d}\right)^{n}$ where no two of the $n$ coordinates in $\mathbb{Z}^{d}$ coincide, with reflecting boundary conditions, it is not hard to see we have expansions $\sum_{|A|=n}\left[P_{t}^{(n)} \tilde{V}_{A}\right]^{2}=t^{-n d / 2}\left(c_{1}^{n}+c_{2}^{n} t^{-1}+c_{3}^{n} t^{-2}+\cdots\right)$. The coefficients $c_{j}^{n}$ are not easy to compute except for $n=1$ or $j=1$. On the other hand, for the theorem we only need $c_{1}^{1}, c_{1}^{2}$ in $d=1, c_{1}^{1}, c_{2}^{1}, c_{1}^{2}$ in $d=2$, and $c_{1}^{1}, c_{2}^{1}$ in $d \geq 3$.

The $n=1$ case is straightforward since $P_{t}^{(1)}$ corresponds to a random walk on $\mathbb{Z}^{d}$. Let's call $f_{x}=\tilde{V}_{x}$ and let $\hat{f}(\xi)=\sum_{x \in \mathbb{Z}^{d}} f_{x} e^{i \xi \cdot x}$. Parseval's identity reads $\sum_{x}\left|f_{x}\right|^{2}=$ $(2 \pi)^{-d} \int_{[-\pi, \pi)^{d}}|\hat{f}(\xi)|^{2} d \xi$. If $\Delta f_{x}=\sum_{y \sim x}\left(f_{y}-f_{x}\right)$ then $\widehat{\Delta f}(\xi)=-q(\xi) \hat{f}(\xi)$ where $q(\xi)=-\sum_{y \sim 0}\left(e^{i \xi \cdot y}-1\right)$. Hence $\sum_{x}\left|e^{t \Delta} f_{x}\right|^{2}=(2 \pi)^{-d} \int_{[-\pi, \pi)^{d}} e^{-2 t q(\xi)}|\hat{f}(\xi)|^{2} d \xi$. Let $\tilde{q}(z)=q(z)-|z|^{2}$. After change of variables we have

$$
t^{d / 2} \sum_{x}\left|e^{t \Delta} f_{x}\right|^{2}=(2 \pi)^{-d} \int_{[-\sqrt{t} \pi, \sqrt{t} \pi)^{d}} e^{-2|z|^{2}} e^{-2 t \tilde{q}(z / \sqrt{t})}\left|\hat{f}\left(\frac{z}{\sqrt{t}}\right)\right|^{2} d z .
$$


Expand $\left|\hat{f}\left(\frac{z}{\sqrt{t}}\right)\right|^{2}=|\hat{f}(0)|^{2}+t^{-1 / 2} D^{1}|\hat{f}|^{2}(0) \cdot z+t^{-1} D^{2}|\hat{f}|^{2}(0) z \cdot z+D^{3}|\hat{f}|^{2}(0) z \otimes z \otimes$ $z+O\left(t^{-2}\right)$ and $e^{-2 t \tilde{q}\left(\frac{z}{\sqrt{t}}\right)}=1-t^{-1}\left(\frac{1}{2} \sum_{i \neq j} z_{i}^{2} z_{j}^{2}-\frac{1}{12} \sum_{i} z_{i}^{4}\right)+O\left(t^{-2}\right)$. We have $t^{d / 2} \sum_{x}\left|e^{t \Delta} f_{x}\right|^{2}=(8 \pi)^{-d / 2}|\hat{f}|^{2}(0)+t^{-1}(8 \pi)^{-d / 2}\left[\frac{1}{4} \Delta|\hat{f}|^{2}(0)-2^{-5}\left(d^{2}-3 d\right)|\hat{f}|^{2}(0)\right]+O\left(t^{-2}\right)$.

Here $\Delta$ is the continuum Laplace operator. Now note that

$$
\bar{V}^{\prime}(\rho)=\sum_{x} E_{\nu_{\rho}}\left[V(\eta)\left(\frac{\eta_{x}-\rho}{\rho(1-\rho)}\right)\right]=\chi(\rho)^{-1 / 2} \sum_{x} \tilde{V}_{x} .
$$

Since $\sum_{x} V_{x}=\hat{f}(0)$ this gives

$$
c_{1}^{1}=\frac{\chi(\rho)}{(8 \pi)^{d / 2}}\left|\bar{V}^{\prime}(\rho)\right|^{2} .
$$

Now $\Delta|\hat{f}|^{2}(0)=-2\left[\sum_{j=1}^{d} \sum_{x}\left|x_{j}\right|^{2} f_{x}+\left(\sum_{x} x_{j} f_{x}\right)^{2}\right]$. This gives

$$
c_{2}^{1}=-\frac{1}{(8 \pi)^{d / 2}}\left[\frac{1}{2} \sum_{j=1}^{d} \sum_{x}\left|x_{j}\right|^{2} \tilde{V}_{x}+\frac{1}{2}\left(\sum_{x} x_{j} \tilde{V}_{x}\right)^{2}+2^{-5}\left(d^{2}-3 d\right)\left|\bar{V}^{\prime}(\rho)\right|^{2}\right] .
$$

The case $n=2$ can be mapped $1 \rightarrow 2$ to a random walk $x_{t}$ on $\left\{x_{1}, x_{2} \in \mathbb{Z}^{d}\right.$ : $\left.x_{1} \neq x_{2}\right\}$. Since $x_{N^{2} t} / N$ converges to Brownian motion on $\mathbb{R}^{2 d}$ with generator the continuum Laplacian $\Delta$, it is not hard to check using the local limit theorem that

$$
t^{d} \sum_{|A|=2}\left|P_{t}^{(2)} \tilde{V}_{A}\right|^{2}=\frac{1}{(8 \pi)^{d}}\left|\sum_{|A|=2} \tilde{V}_{A}\right|^{2}+O\left(t^{-1}\right)
$$

Now

$$
\bar{V}^{\prime \prime}(\rho)=2 \chi(\rho)^{-1} \sum_{|A|=2} \tilde{V}_{A}
$$

This gives

$$
c_{1}^{2}=\frac{\chi(\rho)^{2}}{4(8 \pi)^{d}}\left|\bar{V}^{\prime \prime}(\rho)\right|^{2} .
$$

Proof of Theorem 1.2. The proof follows closely the proof for zero-range in sections 2,3 and 4 , so we only give a sketch. As in Corollary 2.4, the decay estimate allows us to reduce the problem to solving for some particular choice of $V$. In $d=1,2$ for $\bar{V}(\rho)=0$, that choice can be $V(\eta)=\eta(0)-\rho$, and the central limit theorem is proved in $[\mathrm{K}]$. The only remaining case is $d=1, \bar{V}(\rho)=\bar{V}^{\prime}(\rho)=0$. We choose as our candidate $V(\eta)=\tilde{\eta}_{\{0,1\}}$. Let $\tilde{E}_{2}$ denote the subsets of $\mathbb{Z}$ of cardinality 2. For $A, B$ in $\tilde{E}_{2}$, let $\tilde{p}_{t}^{2}(A, B)$ be the solution of

$$
\frac{\partial \tilde{p}^{2}}{\partial t}=\tilde{L}_{2} \tilde{p}, \quad \tilde{p}_{0}^{2}(A, B)=\delta(A, B)
$$

where $\delta(A, B)=1$ if $A=B$ and 0 otherwise. Then $\tilde{g}_{\lambda}^{2}(A)=\int_{0}^{\infty} e^{-\lambda t} \tilde{p}_{t}^{2}(\{0,1\}, A) d t$ is the solution of

$$
\left(\lambda-\tilde{L}_{2}\right) \tilde{g}_{\lambda}^{2}(A)=\delta(A,\{0,1\}) .
$$


Define

$$
G_{\lambda}(\eta)=\sum_{|A|=2} \tilde{g}_{\lambda}^{2}(A) \tilde{\eta}_{A}
$$

Then

$$
(\lambda-L) G_{\lambda}=\tilde{\eta}_{\{0,1\}}
$$

Hence

$$
\frac{1}{\sqrt{N \log N}} \int_{0}^{N t} \tilde{\eta}_{\{0,1\}} d s=M_{t}^{N}+R_{t}^{N}
$$

where $M_{t}^{N}$ and $R_{t}^{N}$ are as in (4.1) and (4.2). We have $\left\|G_{\lambda}\right\|_{L^{2}\left(\nu_{\rho}\right)}^{2}=\operatorname{Var}_{\nu_{\rho}}(\eta(0))\left\|\tilde{g}_{\lambda}^{2}\right\|_{\ell^{2}\left(\mathbb{Z}^{d}\right)}^{2}$ and $\left\|\hat{G}_{\lambda}\right\|_{L^{2}\left(\nu_{\rho}\right)}^{2}=\operatorname{Var}_{\nu_{\rho}}(\eta(0))\left\|\tilde{g}_{\lambda}^{2}\right\|_{\ell^{2}\left(\mathbb{Z}^{d}\right)}^{2}$ and the same estimate as before, $\left\|\tilde{g}_{\lambda}^{2}\right\|_{\ell^{2}\left(\tilde{E}_{2}\right)}^{2} \sim$ $\lambda^{-1}$, gives $E_{P_{\rho}}\left[\left(R_{t}^{N}\right)^{2}\right] \leq C / \log N$ and then Corollary 2.3 implies that $R_{t}^{N}$ converges to the trivial process $X_{t} \equiv 0$. As in section 4 , Lemma 4.1 implies that $M_{t}^{N}$ converge to Brownian motion. The explicit covariance is obtained from Theorem 6.1.

\section{REFERENCES}

[A] ANDJEL E., Invariant measures for the zero-range processes, Ann. Prob., 10 (1982), pp. 525547.

[CG] Cox, T., Griffeath, D., Large deviations for Poisson systems of independent random walks, Z. Wahr. verw. Geb., 66 (1984), pp. 543-558.

[JLQY] Janvresse, E., Landim, C., Quastel, J., Yau, H.T., Relaxation to equilibrium of conservative dynamics I: Zero-range processes, Ann. Probab., 27 (1999), pp. 325-360.

[K] KIPNIs, C., Fluctuations des temps d'occupation d'un site dans l'exclusion simple symetrique, Ann. Inst. Henri Poincare, 23 (1987), pp. 21-35.

[K] KIPNIs, C., Central limit theorems for infinite series of queues and applications to simple exclusion, Ann. Probab., 14 (1986), pp. 397-408.

[KV] Kipnis, C., Varadhan, S.R.S., Central limit theorem for additive functionals of reversible Markov processes and applications to simple exclusions, Comm. Math. Phys., 104 (1986), pp. $1-19$.

[L] LAWLER, G., Intersections of random walks, Birkhauser, Boston, 1991.

[LQY] Janvresse, E., Landim, C., Quastel, J., Yau, H.T., Relaxation to equilibrium of conservative dynamics II: Lattice gases, in preparation.

[LSV] Landim, C., Sethuraman, S., Varadhan, S.R.S., Spectral gap for zero range dynamics, Ann. Probab., 24, pp. 1871-1902.

[R] Rebolledo, R., Remarques sur la convergence en loi de martingales vers des martingales continues, C.R. Acad. Sci. Paris., 285 (1977), pp. 517-520.

[S] Sethuraman, S., Central limit theorems for additive functionals of the simple exclusion process, Ann. Probab., 28 (2000), pp. 277-302.

[Sp] Spitzer, F., Interaction of Markov processes, Adv. Math., 5 (1970), pp. 142-154.

[SV] Stroock, D., Varadhan, S. R. S., Multidimensional diffusion processes, Grundlehren der Mathematischen Wissenschaften 233. Springer-Verlag, Berlin-New York, 1979.

[SX] Sethuraman, S., Xu, L., A central limit theorems for reversible exclusion and zero-range particle systems, Ann. Probab., 24 (1996), pp. 1842-1870. 